\title{
Early Pleistocene Glacial Cycles and the Integrated Summer Insolation Forcing
}

\section{Citation}

Huybers, Peter J. 2006. Early Pleistocene glacial cycles and the integrated summer insolation forcing. Science 313(5786): 508-511.

\section{Published Version}

http://dx.doi.org/10.1126/science.1125249

\section{Permanent link}

http://nrs.harvard.edu/urn-3:HUL.InstRepos:3382981

\section{Terms of Use}

This article was downloaded from Harvard University's DASH repository, and is made available under the terms and conditions applicable to Other Posted Material, as set forth at http:// nrs.harvard.edu/urn-3:HUL.InstRepos:dash.current.terms-of-use\#LAA

\section{Share Your Story}

The Harvard community has made this article openly available.

Please share how this access benefits you. Submit a story.

Accessibility 


\title{
Science MIAAAS
}

\author{
Early Pleistocene Glacial Cycles and the Integrated \\ Summer Insolation Forcing \\ Peter Huybers, et al. \\ Science 313, 508 (2006); \\ DOI: $10.1126 /$ science.1125249
}

\section{The following resources related to this article are available online at www.sciencemag.org (this information is current as of January 5, 2007):}

Updated information and services, including high-resolution figures, can be found in the online version of this article at:

http://www.sciencemag.org/cgi/content/full/313/5786/508

Supporting Online Material can be found at: http://www.sciencemag.org/cgi/content/full/1125249/DC1

A list of selected additional articles on the Science Web sites related to this article can be found at:

http://www.sciencemag.org/cgi/content/full/313/5786/508\#related-content

This article cites 12 articles, 5 of which can be accessed for free:

http://www.sciencemag.org/cgi/content/full/313/5786/508\#otherarticles

This article has been cited by 1 article(s) on the ISI Web of Science.

This article appears in the following subject collections:

Atmospheric Science

http://www.sciencemag.org/cgi/collection/atmos

Information about obtaining reprints of this article or about obtaining permission to reproduce this article in whole or in part can be found at:

http://www.sciencemag.org/help/about/permissions.dtl 


\section{Early Pleistocene Glacial Cycles and the Integrated Summer Insolation Forcing}

\section{Peter Huybers}

Long-term variations in Northern Hemisphere summer insolation are generally thought to control glaciation. But the intensity of summer insolation is primarily controlled by 20,000-year cycles in the precession of the equinoxes, whereas early Pleistocene glacial cycles occur at 40,000-year intervals, matching the period of changes in Earth's obliquity. The resolution of this 40,000 -year problem is that glaciers are sensitive to insolation integrated over the duration of the summer. The integrated summer insolation is primarily controlled by obliquity and not precession because, by Kepler's second law, the duration of the summer is inversely proportional to Earth's distance from the Sun.

A link between changes in glacial extent and Earth's orbital configuration was apparently first proposed by Adhémar $(1,2)$, who postulated that the Antarctic ice sheet exists because the Southern Hemisphere winter is 8 days longer than the Northern one. In this case, winter is the period between the equinoxes. This difference in duration follows from Kepler's second law and from the fact that Earth's closest approach to the Sun, that is, perihelion, currently occurs during Northern Hemisphere winter. Croll modified this hypothesis, arguing that the decrease in insolation associated with being further from the Sun leads to glaciation $(2,3)$. Milankovitch, in turn, argued that summer insolation determines glaciation (4). More recently, once proxies of past glaciation showed that late Pleistocene glacial cycles occurred at $\sim 100,000$-year (100-ky) intervals (5), the amplitude envelope of the precession (i.e., the eccentricity) was identified as accounting for the 100-ky glacial cycles (5-7).

This thread of glacial hypotheses thus implies that precession of the equinoxes controls the occurrence of glacial cycles. Indeed, variations in the intensity of summer insolation are primarily controlled by precession. For example, average insolation on the 21 st day of June at $65^{\circ} \mathrm{N}$ has $80 \%$ of its variance at the precession periods $(1 / 21 \mathrm{ky} \pm 1 / 100 \mathrm{ky})$. The caloric summer half-year at $65^{\circ} \mathrm{N}$, defined as the energy received during the half of the year with the greatest insolation intensity (4), also has more than half its variance in the precession bands. But a major problem exists for the standard orbital hypothesis of glaciation: Late Pliocene and early Pleistocene glacial cycles occur at intervals of $40 \mathrm{ky}(8-11)$, matching the obliquity period, but have negligible 20-ky variability.

One possibility is that the latitudinal gradient in insolation, which enhances obliquity over precession, is more important than local insola-

Department of Earth and Planetary Sciences, Harvard University, Cambridge, MA 02138, USA. E-mail: phuybers@ fas.harvard.edu tion (11). However, models used to explore the effects of changes in the insolation gradient have found that local insolation is the more important control on glacial mass balance (12). Simple models that used summer insolation as the forcing (13-15) exhibited more precessionperiod variability than is observed in the early Pleistocene climate record. Another possibility is that glaciation is controlled by the annual average insolation, which is independent of precession, but this hypothesis requires glacial mass balance to be equally sensitive to winter and summer insolation (16). One climate model (17) that is forced by the complete seasonal cycle
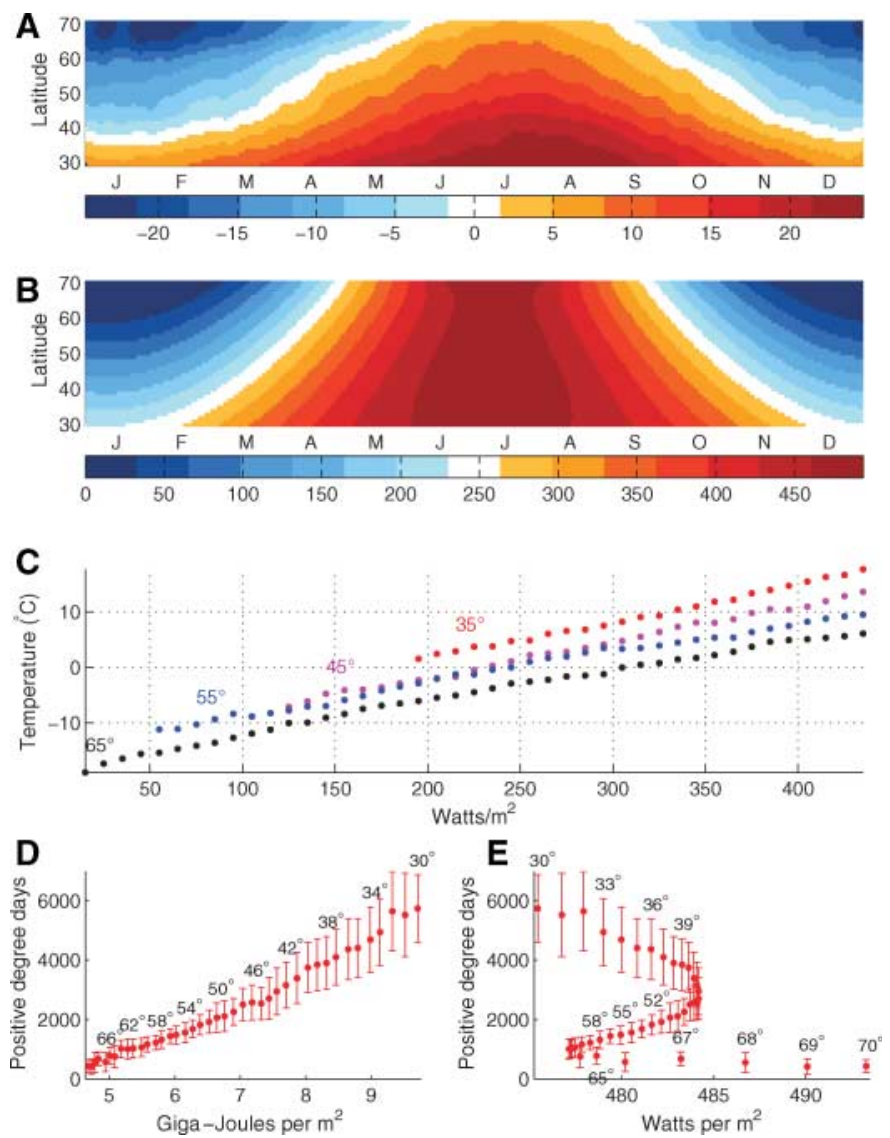

showed predominantly precession-period glacial variability during the early Pleistocene, whereas another, more sophisticated, coupled climate-ice sheet model (18) showed primarily obliquity period variability (although the latter model is for Antarctica near 34 My ago), and neither study identified mechanisms for the differing sensitivities to orbital variations. The origins of strong obliquity over precession-period glacial variability during the early Pleistocene remain unresolved.

Tying insolation at the top of the atmosphere to climate on the ground poses a serious challenge. It is useful to consider empirical relationships between insolation (19) and modern temperature (20). Insolation lagged by 30 days shows an excellent correlation with zonally and diurnally averaged land temperature, $\bar{T}$, for latitude bands north of $30^{\circ} \mathrm{N}\left(r^{2}>\right.$ 0.99 ) (Fig. 1C). Insolation is apparently a good predictor of $\bar{T}$.

A more complicated relationship might have been expected between insolation and $\bar{T}$ when one considers processes such as reflection of radiation by snow, ice, and clouds; changes in heat storage; and the redistribution of heat by the ocean and the atmosphere. The linear relationship between insolation and average temperature does not exclude the importance of these processes but does suggest that their aggregate influence is also correlated with the insolation. Furthermore, the combined heat

Fig. 1. Relationships between insolation and temperatures. (A) Temperature in ${ }^{\circ} \mathrm{C}$ contoured as a function of latitude and month. Temperatures, $\bar{T}$, are diurnal averages from WMO stations and are averaged according to latitude after adjusting for elevation using a lapse rate of $6.5^{\circ} \mathrm{C} / \mathrm{km}$. (B) Insolation at the top of the atmosphere. (C) $\bar{T}$ plotted against insolation for different latitudes $\left(r^{2}>\right.$ 0.99). Latitude bins are $10^{\circ}$, and insolation bins are $10 \mathrm{~W} / \mathrm{m}^{2}$ where insolation has been lagged by 1 month. (D) Positive degree days plotted against summer energy $\left(r^{2}=0.98\right)$. (E) Positive degree days plotted against the intensity of diurnally averaged insolation on June 21st $\left(r^{2}=0.04\right)$. 
transport of the ocean and the atmosphere to latitudes above $30^{\circ} \mathrm{N}$ amounts to $5 \mathrm{PW}$ (petaWatts) (21) and, when spatially averaged, corresponds to $40 \mathrm{~W} / \mathrm{m}^{2}$, or less than $10 \%$ of the summer insolation at the top of the atmosphere at any latitude. In this light, it is reasonable for insolation to primarily control local temperature, particularly during the summer months.

If one accepts the empirical relationship between insolation and temperature, then what is the best measure of insolation's influence on ablation? It is not mean annual insolation: The ablation season is not more than 6 months in duration, and the temperature during the rest of the year seems largely irrelevant (22). Mean summer insolation is a more likely candidate. However, defining summer is difficult because the length of the ablation season should depend on the insolation cycle itself as well as other environmental factors.

A good measure of air temperature's influence on annual ablation is the sum of positive degree days $(22,23)$, defined as $S=\sum_{i} \alpha_{i} T_{i}$, where $T_{i}$ is mean daily temperature on day $i$ and $\alpha$ is one when $T_{i} \geq 0^{\circ} \mathrm{C}$ and zero otherwise. A quantity analogous to $S$ can be defined for insolation. For latitudes between $40^{\circ}$ to $70^{\circ} \mathrm{N}$, the temperature is near $0^{\circ} \mathrm{C}$ when insolation intensity is between 250 and $300 \mathrm{~W} / \mathrm{m}^{2}$ (Fig. 1C), and $\tau=275 \mathrm{~W} / \mathrm{m}^{2}$ is taken as a threshold (24). The number of degree days is postulated to fol- low the sum of the insolation on days exceeding this threshold, $J=\sum_{i} \beta_{i}\left(W_{i} \times 86,400\right)$, where $J$ is termed the summer energy and is measured in joules. $W_{i}$ is mean insolation in $\mathrm{W} / \mathrm{m}^{2}$ on day $i$, and $\beta$ equals one when $W_{i} \geq \tau$ and zero otherwise. Note that ablation responds to both radiative transfer and heat flux from the atmosphere into the ice, but this distinction is not made because insolation and temperature are strongly correlated.

$S$, computed by using $\bar{T}$, monotonically decreases from 6000 at $30^{\circ} \mathrm{N}$ to 400 at $70^{\circ} \mathrm{N}$. The summer energy also steadily decreases toward high latitudes and is highly correlated with the positive degree days $\left(r^{2}=0.98\right)$ (Fig. 1D). In contrast, the average insolation intensity on June 21 st has a more complicated dependence on latitude (owing to the tradeoff between zenith angle and hours of daylight) and has a low correlation with the positive degree days $\left(r^{2}=0.04\right)$ (Fig. 1E). It is perhaps unsurprising that insolation on June 21st fails to correspond to positive degree days. For similar reasons, one would not expect temperature on a single day of the year to adequately predict annual ablation.

Long-term variations in the duration of the summertime and intensity of summer insolation are primarily controlled by the precession of the equinoxes, with more than $80 \%$ of their respective variances within $1 / 21 \mathrm{ky} \pm 1 / 100 \mathrm{ky}$ (Fig. 2, A and B). Duration and intensity are, however, anticorrelated. This is the Achilles' heel of precession control of glaciation: just when Earth is closest to the sun during summer, summertime is shortest. When the intensity is integrated over the summertime, precessionrelated changes in duration and intensity nearly balance one another (25), and the obliquity component is dominant. When $\tau=275 \mathrm{~W} / \mathrm{m}^{2}$, $80 \%$ of the summer energy variance is in the obliquity band (1/41 ky \pm 1/100 ky) (Fig. 2, C and D).

As an example, Earth's orbital configuration when perihelion occurs variously at the equinoxes and at the solstices is shown (Fig. 3) for the interval between 220 and 200 ky ago. When perihelion occurs at summer solstice rather than winter solstice, mean summer insolation at $65^{\circ} \mathrm{N}$ is $54 \mathrm{~W} / \mathrm{m}^{2}$ greater (assuming a fixed obliquity of $23.3^{\circ}$ ), but summer is also 13 days shorter. Changes in the orientation of perihelion with respect to the seasons cause deviations of no more than \pm 0.1 GJ (giga-Joules) from a mean summer energy of 5.0 GJ. In contrast, if perihelion is fixed at summer solstice, an increase in obliquity from $22.1^{\circ}$ to $24.5^{\circ}$ results in an average increase in summer intensity of $24 \mathrm{~W} / \mathrm{m}^{2}$ (Fig. 3C), an increase in summer duration from 133 to 137 days, and an increase in summer energy from 4.9 to $5.3 \mathrm{GJ} / \mathrm{m}^{2}(26)$.

Changes in accumulation, although more difficult to infer from insolation, may also contribute to changes in the glacial mass
Fig. 2. Insolation forcing and Pleistocene glacial variability. (A) Number of days that insolation is above $275 \mathrm{~W} / \mathrm{m}^{2}$ (blue) and the average insolation intensity during this interval (red). Intensity and duration are anticorrelated. (B) Spectral estimate of the duration (blue) and intensity (red), showing that the majority of the variability is at the precession periods. Shaded bands from left to right indicate the 100-ky, 41-ky (obliquity), and 21-ky (precession) bands. (C) Summer energy (red) and the time rate of change of $\delta^{18} O$ (black) for the early Pleistocene and (D) the corresponding spectral estimates. Positive rates of change indicate decreasing ice volume. Variability in both records is predominantly at the 41-ky obliquity period. (E and F) Same as (C) and (D) but for the late Pleistocene. The time rate of change of $\delta^{18} 0$ has variability at the 100 -ky period not present in the forcing.
A

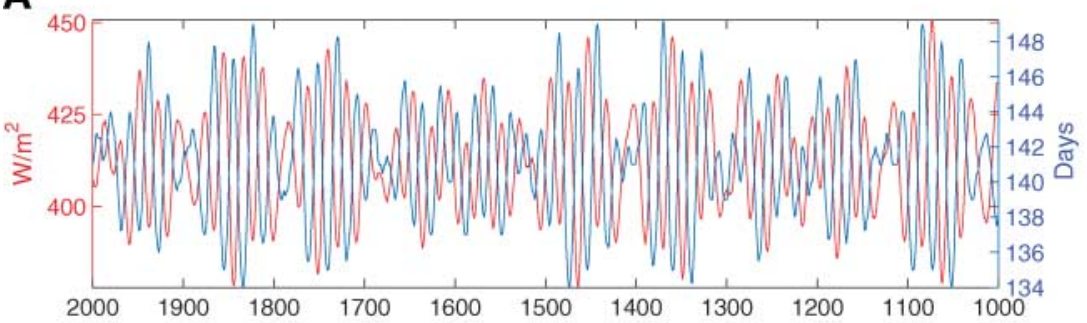

C

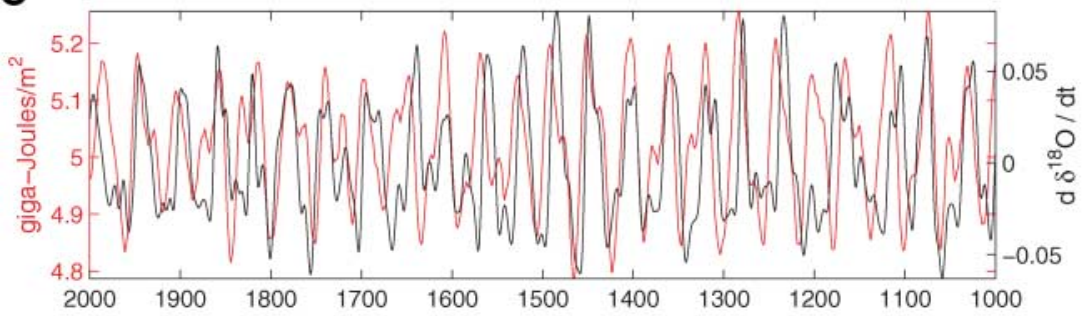

E

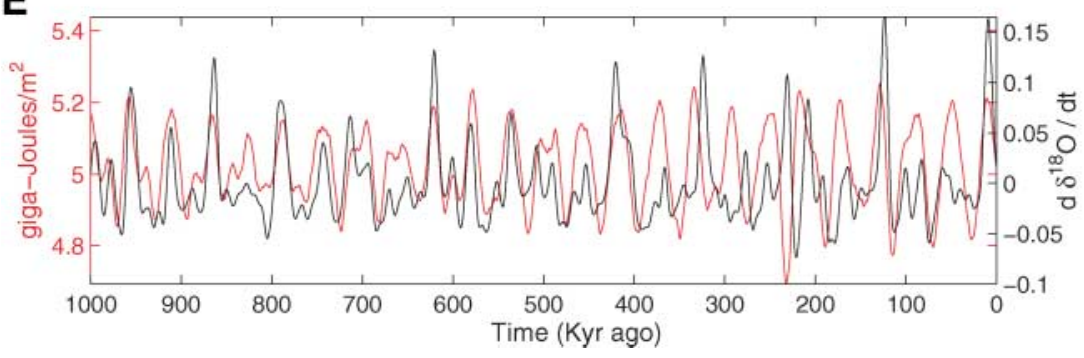

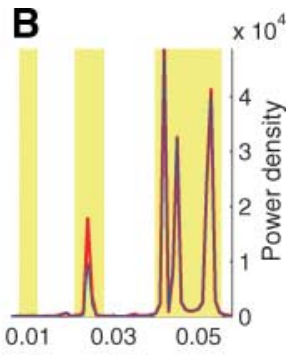

D

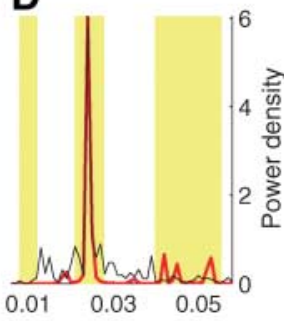

$\mathbf{F}$

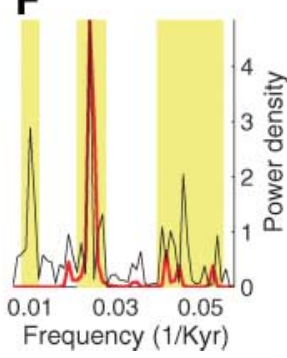


balance. In addition to high-latitude summer energy increasing when obliquity is large, winter energy decreases, possibly decreasing winter temperature and causing a decrease in atmospheric moisture and glacial accumulation.

So far, only modern observations have been used to argue that summer energy is a better indicator of glacial variability than insolation intensity. It remains to test this result against past glacial variations. Changes in summer energy are expected to correspond to rates of ablation and thus are most directly compared against rates of ice volume change (27). After smoothing using an 11-ky tapered window, the time derivative of a composite $\delta^{18} \mathrm{O}$ record is used as a proxy for ice volume change (28). Importantly, the age model for the proxy record does not rely upon orbital assumptions.

There is an excellent correspondence between summer energy at $65^{\circ} \mathrm{N}$ and the rate of ice volume change. For the early Pleistocene, $70 \%$ of the variance in the rate of ice volume change is concentrated at the obliquity band $(1 / 41 \mathrm{ky} \pm$ $1 / 100 \mathrm{ky}, P=0.01$ ) (Fig. 2, C and D), and the obliquity band is in phase and $80 \%$ coherent with the summer energy $(P=0.01)$. There is also a significant correlation between the amplitude of the summer energy forcing and the amplitude of ablation $\left(r^{2}=0.5, P=0.01\right)$, whereas June 21st insolation shows negligible correlation $\left(r^{2}=0.1\right)(29)$. Parametrization of the insolation forcing using summer energy seems to resolve the question of why early Pleistocene glacial cycles occur primarily at 40-ky intervals. More generally, summer energy may explain why obliquity appears to be the primary period of glacial variability throughout the glaciated portions of the Cenozoic (30).

The concept of summer energy also has implications for the $\sim 100$-ky glacial variability during the late Pleistocene (31). Obliquity period variability remains the strongest component of ice volume change during the late Pleistocene, having nearly the same magnitude as during the early Pleistocene but accounting for a smaller fraction of variance $(40 \%)$ because of enhanced precession ( $26 \%$ at $1 / 21 \mathrm{ky} \pm 1 / 100 \mathrm{ky}$ ) and 100 -ky period variance $(22 \%$ at $1 / 100 \mathrm{ky} \pm$ 1/300 ky) (Fig. 2, E and F). Note that the rate of change used here, rather than magnitude of ice volume, has relatively more variance at high frequencies.

Fig. 3. The Earth's variable orbit around the Sun. (A) Earth's orbit when perihelion occurs at Northern Hemisphere summer solstice (red), fall equinox (orange), winter solstice (blue), and spring equinox (light blue), corresponding to the orbital configurations near 220.2, $214.6,209.2$, and 203.5 ky ago, respectively. The eccentricity of Earth's orbit averages 0.05 during this interval. The orbit is to scale and oriented so that spring equinox always occurs at the three o'clock position. March 21st is referenced to the spring equinox, and the location of the Earth is shown every 45.7 days (colored dots with dates given as month/day). Earth moves counterclockwise. The orbit having perihelion during Northern Hemi-
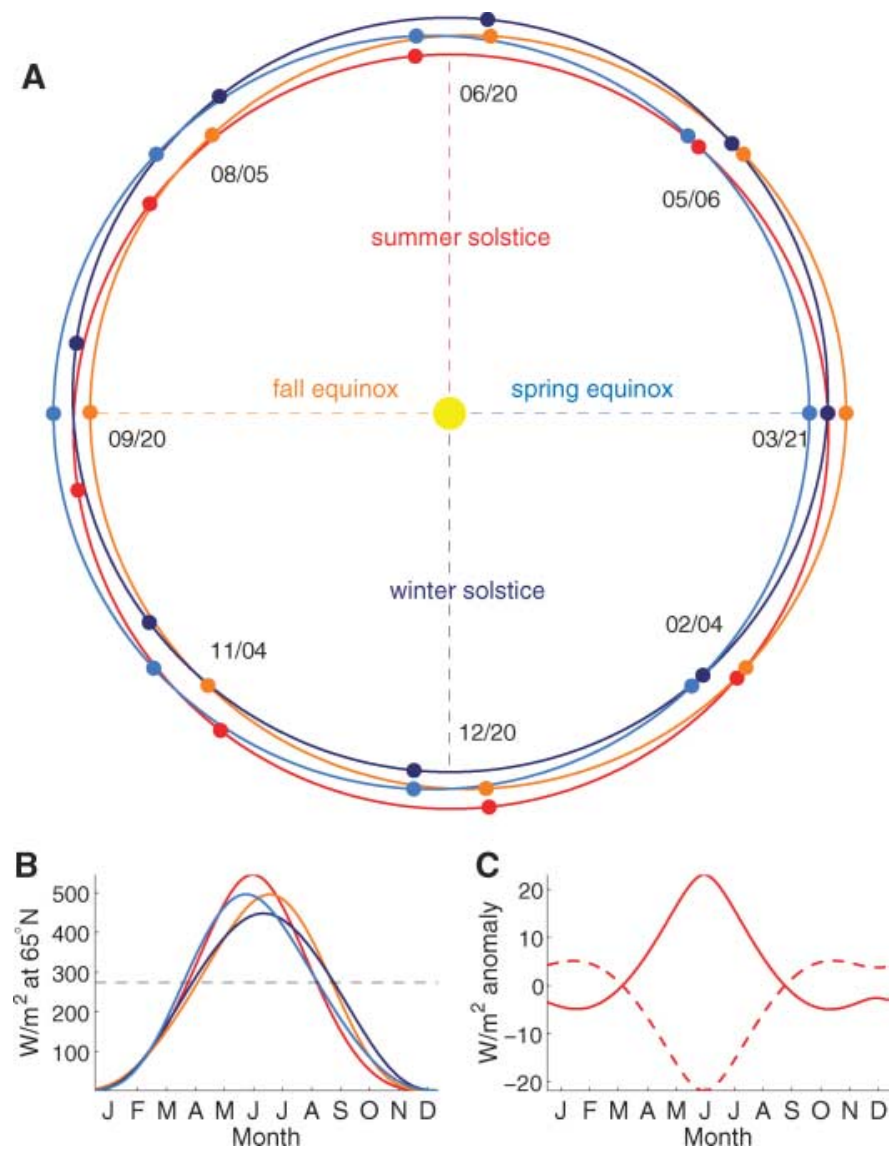

sphere summer (red) reaches fall equinox the soonest. (B) Seasonal variations in insolation at $65^{\circ} \mathrm{N}$. The $x$ axis is labeled with the midpoint of each month. The orbit with perihelion at summer solstice (red) achieves the greatest insolation intensity but also has the shortest duration above a $275 \mathrm{~W} / \mathrm{m}^{2}$ threshold (indicated by the horizontal dashed line). (C) Anomalies in insolation for obliquity values of $22.1^{\circ}$ (dashed) and $24.5^{\circ}$ (solid) relative to a mean obliquity of $23.3^{\circ}$ for the orbit with perihelion at summer solstice.
The amplitude of the summer energy and rates of ablation show less agreement during the late Pleistocene $\left(r^{2}=0.4\right)$ than during the early Pleistocene. The most rapid ablation events, known as terminations, follow periods of greatest ice volume (32), suggesting that the sensitivity to summer energy depends on the amount of ice volume. To quantify this effect, the amount of ice volume is estimated with the use of $\delta^{18} \mathrm{O} 10 \mathrm{ky}$ before peak ablation, and sensitivity is defined as the ratio between the amplitude of ablation and the amplitude of the local maximum in summer energy nearest in time. A significant correlation is observed between ice volume and sensitivity $\left(r^{2}=0.6\right)$. Perhaps large ice sheets are inherently more unstable (13), or perhaps they are more strongly forced by local insolation because they extend to lower latitudes.

A cooling climate during the Pleistocene $(30,33)$ may have permitted ice volume to build up over multiple forcing cycles, allowing sensitivity to increase until an increase in summer energy triggers a glacial termination. In agreement with earlier results (16), terminations occur at intervals of about two (80-ky) or three (120-ky) obliquity cycles, on average giving the $\sim 100$-ky variability. A cooling Pleistocene climate may also be expected to increase the threshold $\tau$, at which melting occurs. A higher $\tau$ makes summer energy more variable and more sensitive to precession variations (fig. S1). For example, raising $\tau$ from 275 to 340 $\mathrm{W} / \mathrm{m}^{2}$ more than doubles the summer energy variance and gives equal precession and obliquity period variability. Thus, a cooling climate and increased $\tau$ may help explain both the larger glacial variations and the appearance of precession period variability during the late Pleistocene.

The hypothesis presented here follows from both Adhémar's argument regarding seasonal duration and Croll and Milankovitch's argument regarding insolation intensity. Taking duration and intensity together, it now appears that summer energy controls early Pleistocene glacial variability. However, the 100-ky glacial cycles of the late Pleistocene have a more complicated relationship with the forcing, and their explanation will require a better understanding of ice sheet-climate interactions.

References and Notes

1. ]. Adhémar, Révolutions de la Mer (Carilian-Goeury et V. Dalmont, Paris, 1842).

2. E. Bard, C. R. Geosci. 336, 603 (2004).

3. J. Croll, Philos. Mag. 28, 121 (1864).

4. M. Milankovitch, Kanon der Erdbestrahlung und seine Andwendung auf das Eiszeitenproblem (Royal Serbian Academy, Belgrade, 1941).

5. J. D. Hays, ]. Imbrie, N. J. Shackleton, Science 194, 1121 (1976)

6. J. Imbrie, J. Z. Imbrie, Science 207, 943 (1980).

7. J. Imbrie et al., Paleoceanography 8, 699 (1993)

8. J. Imbrie et al., Paleoceanography 7, 701 (1992).

9. R. Tiedemann, M. Sarnthein, N. J. Shackleton, Paleoceanography 9, 619 (1994) 
10. L. Lisiecki, M. Raymo, Paleoceanography 20, 10.1029/2004PA001071 (2005).

11. M. Raymo, K. Nisancioglu, Paleoceanography $\mathbf{1 8}$ 10.1029/2002PA000791 (2003).

12. K. Nisancioglu, thesis, Massachusetts Institute of Technology (2004).

13. P. Clark, R. Alley, D. Pollard, Science 286, 1104 (1999).

14. E. Tziperman, H. Gildor, Paleoceanography $\mathbf{1 8}$ 10.1029/2001PA000627 (2003).

15. D. Paillard, Nature 391, 378 (1998).

16. P. Huybers, C. Wunsch, Nature 434, 491 (2005).

17. A. Berger, X. Li, M. Loutre, Quat. Sci. Rev. 18, 1 (1999)

18. R. DeConto, D. Pollard, Palaeogeogr. Palaeoclimatol. Palaeoecol. 198, 39 (2003)

19. A. Berger, M. F. Loutre, Earth Planet. Sci. Lett. 111, 369 (1992).

20. Daily average surface temperatures are estimated by using the network of 8892 World Meteorological Organization (WMO) stations above $30^{\circ} \mathrm{N}$ for the years 1994 to 1999 . All stations that have greater than $80 \%$ data coverage are used. Data gaps are filled by linear interpolation. Stations are standardized to $1 \mathrm{~km}$ of elevation assuming a lapse rate of $6.5^{\circ} \mathrm{C} / \mathrm{km}$, were binned according to $1^{\circ}$ or $10^{\circ}$ latitude bands (as indicated in the text), and are then averaged together. Lastly, each of the six consecutive seasonal cycles are averaged together, yielding average annual cycles as a function of latitude.

21. C. Wunsch, J. Clim. 18, 4374 (2005)

22. W. Paterson, Physics of Glaciers (Pergamon Press, Oxford ed. 3, 1994)

23. R. Braithwaite, Y. Zhang, J. Glaciol. 152, 7 (2000).

24. The use of a constant value for $\tau$ illustrates the concept of summer energy. A more detailed description would take into account that $\tau$ is expected to be spatially and temporally variable, depending on factors such as elevation, albedo, clouds, heat transport, and greenhouse gas concentrations. Note, however, that results are not sensitive to plausible choices of $\tau$ and that values less than $325 \mathrm{~W} / \mathrm{m}^{2}$ yield broadly consistent summer energies (fig. S1). Summer energy values at $65^{\circ} \mathrm{N}$ are given in table S1.

25. The relationship between insolation intensity and insolation energy is more precisely illustrated by noting that $I \propto 1 / r^{2}$, where $I$ is insolation intensity and $r$ is the distance from the Earth to the Sun. Conservation of angular momentum (or, equivalently, Kepler's second law) dictates that $d t \propto r^{2} d \lambda$, where $d t$ is an infinitesimal change in time and $d \lambda$ the corresponding change in solar longitude. The energy received by the Earth is then $]=I d t$ $\propto d \lambda$. In contrast with $I$, the $J$ between any two solar longitudes is independent of $r$ and, thus, independent of the precession of the equinoxes.

26. Are past changes in summer energy sufficient to cause the waxing and waning of ice sheets? Although a full answer requires a realistic model of Pleistocene climate, some indication is provided by modern glacial changes: $\mathrm{A}$ $2.4 \mathrm{~W} / \mathrm{m}^{2}$ global average increase in radiative forcing caused by greenhouse gases (34) has apparently led to a general decrease in glacial mass (35), suggesting that glaciers are sensitive to relatively small changes in the radiation budget.

27. C. Wunsch, Clim. Dyn. 20, 353 (2003)

28. Materials and Methods are available as supporting material on Science Online.

29. Amplitude cross correlation was computed by pairing local maxima in insolation with the nearest (in time) maximum in the rate of change of ice volume. Before identifying maxima, both the $\delta^{18} \mathrm{O}$ record and the summer energy were smoothed by using an 11-ky tapered window. There are 34 local maxima in summer energy at $65^{\circ} \mathrm{N}$ between 2 and $1 \mathrm{My}$ ago and another 34 between $1 \mathrm{My}$ ago and the present. Squared cross correlations of 0.4 and higher have $P$ values of less than 0.01 . Spectral and coherence analysis is performed by using Thomson's multitaper method (36).
30. J. C. Zachos, N. J. Shackleton, ]. S. Revenaugh, H. Pälike, B. P. Flower, Science 292, 274 (2001).

31. Similar with the early Pleistocene, late Pleistocene ice volume change has significant variability concentrated at the obliquity band, which is in phase and coherent with summer energy $(P=0.01)$. That the obliquity component of summer energy varies symmetrically between the hemispheres helps explain the symmetry of glacial variations between the hemispheres. Also, the increase in summer energy near 420 ky ago, absent in measures of summer insolation forcing relying on intensity, helps explain the corresponding glacial termination.

32. M. E. Raymo, Paleoceanography 12, 577 (1997).

33. M. Raymo, Annu. Rev. Earth Planet. Sci. 22, 353 (1994)

34. ]. Houghton et al., Eds., Climate Change 2001: The Scientific Basis. Contribution of Working Group I to the Third Assessment Report of the Intergovernmental Panel on Climate Change (Cambridge Univ. Press, New York, 2001)

35. ]. Oerlemans, Science 308, 675 (2005); published online 3 March 2005 (10.1126/science.1107046).

36. D. Percival, A. Walden, Spectral Analysis for Physical Applications (Cambridge Univ. Press, Cambridge, 1993).

37. This paper benefited from discussion with E. Boyle, B. Curry, M. Raymo, P. Stone, E. Tziperman, and C. Wunsch. J. Levine provided valuable assistance in calculating the insolation. The NSF paleoclimate program supported this work under grant no. ATM-0455470.

\section{Supporting Online Material}

www.sciencemag.org/cgi/content/full/1125249/DC1

SOM Text

Figs. S1 to S3

References

23 January 2006; accepted 9 June 2006

Published online 22 June 2006;

$10.1126 /$ science. 1125249

Include this information when citing this paper.

\section{A Thick Cloud of Neptune Trojans and Their Colors}

\section{Scott S. Sheppard ${ }^{1 *}$ and Chadwick A. Trujillo ${ }^{2}$}

The dynamical and physical properties of asteroids offer one of the few constraints on the formation, evolution, and migration of the giant planets. Trojan asteroids share a planet's semimajor axis but lead or follow it by about $60^{\circ}$ near the two triangular Lagrangian points of gravitational equilibrium. Here we report the discovery of a high-inclination Neptune Trojan, $2005 \mathrm{TN}_{53}$. This discovery demonstrates that the Neptune Trojan population occupies a thick disk, which is indicative of "freeze-in" capture instead of in situ or collisional formation. The Neptune Trojans appear to have a population that is several times larger than the Jupiter Trojans. Our color measurements show that Neptune Trojans have statistically indistinguishable slightly red colors, which suggests that they had a common formation and evolutionary history and are distinct from the classical Kuiper Belt objects.

$\mathrm{T}$ he Neptune Trojans are only the fourth observed stable reservoir of small bodies in our solar system; the others are the Kuiper Belt, main asteroid belt, and jovian Trojans. The Trojan reservoirs of the giant planets lie between the rocky main belt asteroids and the volatile-rich Kuiper Belt. The effects of nebular gas drag (1), collisions (2), planetary

\footnotetext{
${ }^{1}$ Department of Terrestrial Magnetism, Carnegie Institution of Washington, 5241 Broad Branch Road NW, Washington, DC 20015, USA. ${ }^{2}$ Gemini Observatory, 670 North A'ohoku
} Place, Hilo, HI 96720, USA.

*To whom correspondence should be addressed. E-mail: sheppard@dtm.ciw.edu migration $(3,4)$, overlapping resonances $(5,6)$, and the mass growth of the planets $(7,8)$ all potentially influence the formation and evolution of the Neptune Trojans. The number of Jupiter Trojans is comparable to the main asteroid belt (9). One Neptune Trojan was discovered serendipitously in 2001 (10). Our ongoing dedicated Trojan survey has found three additional Neptune Trojans (Table 1).

Stable minor planets in the triangular Lagrangian Trojan regions, called the leading L4 and trailing L5 points, are said to be in a $1: 1$ resonance with the planet because each completes one orbit about the Sun with the period of the parent planet. The Neptune Trojans are distinctly different from other known Neptune resonance populations found in the Kuiper Belt. Kuiper Belt resonances such as the 3:2 (which Pluto occupies) and 2:1 may owe their existence to sweeping resonance capture of the migrating planets (11). The Neptune Trojans, however, would be lost because of migration and are not captured during this process $(3,4,10)$.

Numerical dynamical stability simulations have shown that Neptune may retain up to $50 \%$ of its original Trojan population over the age of the solar system after any marked planetary migration $(4,12)$. These simulations also demonstrate that Saturn and Uranus are not expected to have any substantial primordial Trojan populations. Recent numerical simulations of small bodies temporarily passing through the giant planet region, such as Centaurs, have shown that Neptune cannot currently efficiently capture Trojans even for short periods of time $(4,13)$. Thus, capture or formation of the Trojans at the Lagrangian regions likely occurred during or just after the planet formation epoch, when conditions in the solar system were vastly different from those now. We numerically integrated (14) several orbits similar to each of the known Neptune Trojans and found that the majority of test particles near each known Neptune Trojan were stable over the age of the solar system.

Various mechanisms have been proposed that dissipated asteroid orbital energy to perma- 